\title{
Body Weight Changes in Hyperthyroidism: Timing and Possible Explanations during a One Year Repeated Measurement Study
}

\author{
Jesper Karmisholt ${ }^{a, b} \quad$ Allan Carléa, Stig Andersen $^{\mathrm{b}, c}$ \\ ${ }^{a}$ Department of Medical Endocrinology, Aalborg University Hospital, Aalborg, Denmark; ${ }^{b}$ Clinical Institute, Aalborg \\ University, Aalborg, Denmark; ' Department of Geriatrics, Aalborg University Hospital, Aalborg, Denmark
}

\section{Keywords}

Hyperthyroidism · Repeated measurements · Activity of daily living - Exercise capacity · Cardiopulmonary exercise testing · Body weight · Body composition · Antithyroid drug treatment

\begin{abstract}
Background: Weight gain during treatment of hyperthyroidism is a frequent and for many patients unwanted outcome. With this repeated measurement study, we explored the timing of weight changes during the first year of antithyroid drug (ATD) treatment and assessed the correlation between body weight changes and changes in thyroid hormones, resting energy expenditure (REE), physical activity level, and energy efficiency. Methods: Patients with new onset hyperthyroidism were investigated every second month during the first year of ATD treatment. At each investigation, the following were measured: body weight, thyroid hormone concentrations, physical activity level, and daily number of steps, REE, and exercise performance. Results: Two men and eleven women, all sedentary, mean age 49(SD: 9.3) years were included. Significant changes after 1 year occurred for body weight (68.9-74.1 kg), thyroid hormones
\end{abstract}

karger@karger.com www.karger.com/etj

C) 2020 European Thyroid Association Published by S. Karger AG, Basel

Karger"

(free T3 [fT3] 17.5 to $4.42 \mathrm{pmol} / \mathrm{L}), \mathrm{REE}(1,630-1,484 \mathrm{kcal} / 24$ h), and energy efficiency at lower (50 W) workloads (16.0$17.6 \%)$. In individual patients, only REE and fT3 correlated to changes in body weight. Physical activity level did not change during treatment. Conclusion: In this study, treatment of hyperthyroidism was associated with marked increase in body weight in the patients. This increase correlated to a decrease in REE and only to a negligible extent to changes in energy efficiency and not at all to changes in physical activity level of daily living.

(c) 2020 European Thyroid Association Published by S. Karger AG, Basel

\section{Introduction}

Patients with untreated hyperthyroidism experience a plethora of symptoms of which weight loss is frequent [1], and most patients experience weight gain when the disease is treated $[2,3]$. The level of resting energy expenditure (REE) in the untreated disease and the changes during treatment is considered the main driver of the weight alteration [4]. However, the untreated hyperthyroid patients are characterized by reduced exercise capacity 
which improves after 1 year of treating the disease $[5,6]$. In addition, complaints of muscle weakness and muscle pain, and changes in skeletal muscle function have been documented in patients with hyperthyroidism [7-9]. These changes may negatively affect the daily activity level in patients with hyperthyroidism.

A reduction in physical activity level may offset some of the weight loss in the untreated state, as physical activity level is another factor with impact on body weight. The physical activity level of daily living in untreated hyperthyroidism has been only scarcely studied [10], and changes during treatment have only been studied in rats [11], which have very pronounced stray behavior compared to humans.

In this prospective repeated measurement study, we aimed to explore the timing of changes in body weight and its correlation to REE, cardiopulmonary exercise performance, and physical activity of daily living, in patients with newly diagnosed hyperthyroidism who received antithyroid drug (ATD) treatment for 1 year.

\section{Materials and Methods}

Consecutive patients with newly diagnosed hyperthyroidism (suppressed thyrotropin and elevated thyroxine in serum) referred to our unit by their general practitioner were included. Exclusion criteria were previous history of thyroid disease, medical treatment for thyroid disease for more than 1 week, use of medication which could explain the abnormal thyroid function tests, presence of a psychiatric diagnosis or physical incapacity, pregnancy within the last 12 months, and age below 18 or above 80 years.

Thirteen hyperthyroid patients were included, eleven women and 2 men, eleven had Graves' disease, and 2 had multinodular goiter. This study was approved by the Regional Ethics Committee in North-Jutland and Viborg County, Denmark. Informed consent was signed by the patients before entering the study.

The patients were tested at baseline and every second month for 12 months. Each of the 7 visits included physical examination, measurement of REE, and cardiopulmonary exercise testing on a bike-cycle, and assessment of activity of daily living and blood samplings. The participants attended the investigational sessions after an overnight fast. Blood samples were drawn between 08:30 and 13:00 h, and serum was separated and frozen at $-20^{\circ} \mathrm{C}$ immediately after the investigation.

\section{Clinical Investigation}

The patients were weighed (digital weight, SECA, Kolding, Denmark) in light clothing, without shoes. Blood pressure and heart rate (Omron M4-I, Matsusaka, Japan) were measured after $30 \mathrm{~min}$ of resting in the supine position. At baseline, the patients were asked to estimate their weight 6 months prior to disease presentation, a weight termed premorbid weight.

Height measurement (Harpenden Stadiometer, Holtain Ltd., Crosswell, UK) was performed when the body composition was measured with dual X-ray absorptiometry (DXA). Whole-body DXA (Discovery A, Hologic Inc., Bedford, MA, USA) scan was performed at inclusion and after 1 year of treatment.

\section{Resting Energy Expenditure and Cardiopulmonary Exercise} Testing

REE was measured using indirect calorimetry (Deltatrac II Metabolic Monitor, Datex, Helsinki, Finland) on the resting patient in the supine position placed under a ventilated hood. Measurements were performed every minute, and the last twenty of thirty measurements were used for calculations. REE was expressed as kilocalories spent/24 h and as REE/“expected REE," where "expected REE" was calculated according to the HarrisBenedict formula [12]. An ergometer cycle (Ergonoline Via Sprint 150p, Bitz, Germany) linked to and controlled via a PC to an Oxycon Pro (Jaeger, Wurzburg, Germany) metabolic monitor was used for the CPET. Energy efficiency was calculated as the ratio between the energy expenditure measured based on the oxygen consumption versus the external work performed on the bike. Energy efficiency was calculated at 50 and 75 Watt (EFFE 50 and $75 \mathrm{~W}$ ) and at the maximum workload. The exercise started with a load of $10 \mathrm{~W}$ and the load increased stepwise with $1 \mathrm{~W}$ every $5 \mathrm{~S}$ until $50 \mathrm{~W}$ and further again until $75 \mathrm{~W}$. At the 50 and $75 \mathrm{~W}$ levels, the load was unchanged for $90 \mathrm{~s}$, and blood pressure, heart rate, and a BORG scale [13] assessing the perception of physical exertion was used. After $75 \mathrm{~W}$, the load increased with $1 \mathrm{~W}$ every $5 \mathrm{~S}$, and the patients exercised until they felt uncomfortably tired, dyspneic, or unpleasant in other ways. After this, the load was reduced to $30 \mathrm{~W}$, and the patients were asked to pedal at their own pace a couple of minutes before stopping. During the exercise, the patient was connected via a facial mask to the Oxycon Pro monitor which measured respiratory flow rate and oxygen and carbon dioxide concentration breath-by-breath. All patients were familiar with bike cycling, and yet, 1 patient was not able to perform the exercise test. Both the Deltatrac metabolic monitor and the Oxycon Pro were calibrated as recommended by the manufacturer.

\section{Physical Activity in Daily Living}

At each investigation, the patients answered a questionnaire regarding their level of daily physical activity [14]. In this questionnaire, the patients report how many hours per day they were occupied with different activities and data were subsequently transformed to metabolic equivalents (METs) of task or METs per day, as shown in Table 3. A MET value of 1 roughly corresponds to the energy expenditure at rest or the REE [15]. In addition, the number of steps taken, 3 consecutive workdays between each investigation, was measured. The steps were measured with a pedometer (Yamax SW-200, Yamax Corp., Tokyo, Japan) with the counter window covered. Three separate pedometers were used, and the median number of steps for the 3 measurement days is reported.

Assays

Measurements of thyrotropin (TSH), free T4 (fT4), and free T3 (fT3) were performed with an Electro-Chemi Luminescence Immuno Assay method on Modular Analytics E170 (Roche, Mannheim, Germany). Assay characteristics with detection limit and reference ranges given by the manufacturer were TSH, 0.005 and $0.27-4.2 \mathrm{mU} / \mathrm{L}$; fT4, 0.3 and $12-22 \mathrm{pmol} / \mathrm{L}$; and fT3, 0.4 and 3.1-6.8 pmol/L. Hormones were measured in random order and as duplicates. Measured coefficient of variation (CV) (and manu- 
facture reported single-run CV) for the TSH, fT4, and fT3 assays were 0.77 (1.8), 0.84 (1.3), and 1.2 (1.9) \%, respectively. TRAb was measured using a receptor assay (DYNOtest TRAK, Thermo Fisher, Berlin, Germany).

\section{Statistics}

For comparison between paired data, paired $t$ test was used. For associations, Pearson correlation coefficient was used, and for prediction, regression analyses were used. For calculation on categorical data, $\chi^{2}$ or Fisher's exact test was used. Within-person correlations were calculated using ANCOVA as recommended $[16,17]$. For between-person correlation, we performed a between-investigation correlation in which a correlation between 2 variables was calculated to investigate if high (or low) values in 1 variable correlated to high (or low) values in the other at a group level. For this analysis, Pearson correlation on mean values was performed as recommended for between-person analyses [18].

A $p$ value of $<0.05$ was considered significant. The Statistical Package for the Social Sciences version 26.0 (SPSS, Inc, Chicago, IL, USA) and Excel 2003 (Microsoft Corp., Redmond, WA, USA) was used.

\section{Results}

All patients were sedentary and none had atrial fibrillation. The initial symptoms triggering contact with a physician were dyspnea, palpitations, excessive sweating, or tiredness in 11 of the patients. Five were tobacco users. All patients were started on methimazole, and 3 patients received beta-adrenergic blocking agents (propranolol) in the first weeks. Table 1 shows patient characteristics and physical activity measures at baseline and after 1 year.

\section{Timing of Changes}

fT3 was within reference limits after 2 and 4 months in 10 and 12 patients, respectively.

Figure 1 shows the timing of changes in fT3, TSH, body weight, REE, physical activity, number of steps per day, and EFFE 50 and $75 \mathrm{~W}$ as measured every second month after initiation of ATD treatment. The most marked changes occurred during the first 2 months; however, body weight continued to increase up to 8 months after initiation of treatment.

\section{Body Weight and Body Composition}

The mean weight at baseline was 68.9 (SD: 13.1) kg corresponding to a mean BMI of 24.7 (SD: 4.99$) \mathrm{kg} / \mathrm{m}^{2}$. Comparing the weight at presentation with the patientreported premorbid weight showed a mean (SD) weight loss of $2.1(4.0) \mathrm{kg}$, during the 6 months prior to presentation. All, except 1 patient, experienced a weight gain during this study, with a difference in increase between 1 and
Table 1. Patient and exercise characteristics at baseline and after 12 months of ATD treatment of hyperthyroidism

\begin{tabular}{|c|c|c|c|}
\hline & $\begin{array}{l}\text { Baseline } \\
\text { mean (SD) }\end{array}$ & $\begin{array}{l}\text { After } 1 \text { year } \\
\text { mean (SD) }\end{array}$ & $p$ value \\
\hline Age, years & $48.8(9.34)$ & $49.8(9.29)$ & na \\
\hline Sex, men/women & $2 / 11$ & na & na \\
\hline Weight, kg & $68.9(13.1)$ & $74.1(11.2)$ & 0.011 \\
\hline $\mathrm{BMI},{ }^{\S} \mathrm{kg} / \mathrm{m}^{2}$ & $24.7(4.99)$ & $26.6(4.40)$ & 0.011 \\
\hline Heart rate, bpm & $77(2.9)$ & $64(2.8)$ & 0.017 \\
\hline Bone mass, ${ }^{*} \mathrm{~kg}$ & $2.78(0.378)$ & $2.79(0.300)$ & 0.608 \\
\hline Lean mass, ${ }^{*} \mathrm{~kg}$ & $39.2(6.79)$ & $41.9(5.43)$ & 0.066 \\
\hline Fat mass, ${ }^{*} \mathrm{~kg}$ & $29.8(11.4)$ & $28.3(8.52)$ & 0.545 \\
\hline $\mathrm{REE}^{\#}{ }^{*} \mathrm{kcal} / 24 \mathrm{~h}$ & $1,630(257.0)$ & $1,484(243.3)$ & 0.030 \\
\hline REE/expected, ${ }^{* *} \%$ & $115(14.4)$ & $100(12.7)$ & 0.010 \\
\hline $\mathrm{TSH}, \mathrm{mU} / \mathrm{L}$ & $<0.01(0.002)$ & $1.74(1.29)$ & $<0.001$ \\
\hline $\mathrm{fT} 4, \mathrm{pmol} / \mathrm{L}$ & $46.2(25.7)$ & $16.4(2.24)$ & 0.002 \\
\hline $\mathrm{fT} 3, \mathrm{pmol} / \mathrm{L}$ & $17.5(10.6)$ & $4.42(0.723)$ & 0.001 \\
\hline Pedometer, steps/day & $7,212(2,725)$ & $7,857(4,098)$ & 0.564 \\
\hline Daily activity ${ }^{\$}$ METs/day & $40.8(8.64)$ & $42.4(9.42)$ & 0.154 \\
\hline Efficiency ${ }^{\mathcal{E}} 50 \mathrm{~W}, \%$ & $16.0(2.60)$ & $17.6(2.21)$ & 0.033 \\
\hline Efficiency ${ }^{\mathfrak{E}} 75 \mathrm{~W}, \%$ & $20.5(6.1)$ & $20.8(3.4)$ & 0.901 \\
\hline Efficiency ${ }^{\mathfrak{E}} \max$ load, \% & $23.7(8.54)$ & $24.6(8.71)$ & 0.588 \\
\hline Max load, W & 93 (19) & $92(29)$ & 0.980 \\
\hline
\end{tabular}

$\mathrm{SD}$, standard deviation; DXA, dual X-ray absorptiometry; MET, metabolic equivalent; W, watt; fT3, free T3; fT4, free T4; $\mathrm{TSH}$, thyrotropin; REE, resting energy expenditure; max, maximum. ${ }^{\S} \mathrm{BMI}$, weight in kilogram divided by squared height, in meters. ${ }^{*}$ Body composition as measured by DXA. ${ }^{*}$ REE measured in kilocalories per $24 \mathrm{~h} .{ }^{* *} \mathrm{REE} /$ expected - measured REE, divided by the expected REE, based on the Harris-Benedict formula [12]. \$ Daily activity - based on a questionnaire, with output as METs per $24 \mathrm{~h} \mathrm{[14].}{ }^{\mathfrak{E}}$ Efficiency was measured energy expenditure, based on oxygen consumption, divided by the external work performed on the bike. Efficiency was measured when biking at 50 and $75 \mathrm{~W}$ and at maximum load.

$15 \mathrm{~kg}$ and with a significant mean increase for all patients of 5.2 (SD: 6.3 ) kg, $p=0.011$. The 1 patient with decrease in body weight was the heaviest patient, who weighed $105.5 \mathrm{~kg}$ at baseline and 96.6 at the last follow-up investigation. Body weight after 1 year of treatment was on average $4.8(5.5) \mathrm{kg}, p=0.017$, higher than the premorbid weight. Weight loss prior to presentation did not correlate to degree of hyperthyroidism (fT3) at baseline and not to the weight gained, during the study.

Body composition measured by DXA did not show any significant change during 1 year of follow-up (Table 1). On average (SD), change in bone mass/lean mass/fat mass were $-16(84) / 2,957(3,846) / 953(4,241)$ grams, during the study year. However, fT3 at baseline significantly predicted the change in lean mass during treatment, $R^{2}=0.78, p=0.004$. 
Fig. 1. Mean and standard error of mean of various measures at baseline ( 0 months) and at investigations every second month after initiation of ATD treatment in sedentary patients with newly diagnosed hyperthyroidism. Reference limits for fT3 were 3.1-6.8 $\mathrm{pmol} / \mathrm{L}$ and for TSH $0.27-4.2$ $\mathrm{mU} / \mathrm{L}$. fT3, free T3; TSH, thyrotropin; REE, resting energy expenditure; PAS, physical activity measured with a questionnaire; PEDO, number of steps per day; EFFE $50 \mathrm{~W}$, energy efficiency at $50 \mathrm{Watt}$; EFFE $75 \mathrm{~W}$, energy efficiency at $75 \mathrm{Watt}$; ATD, antithyroid drug.
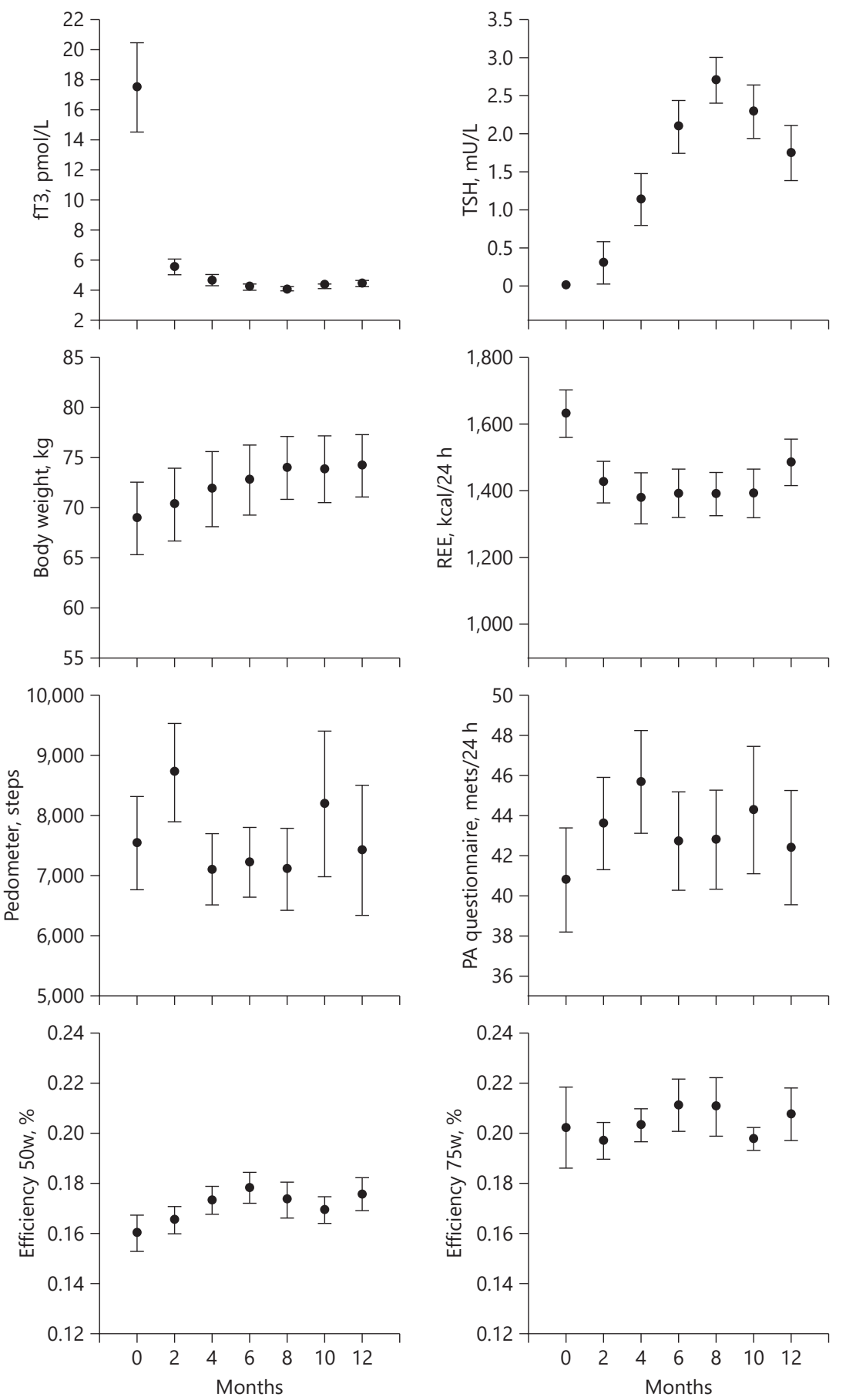

\section{Physical Activity and Exercise Performance}

Daily physical activity level and exercise capacity (maximum load) did not change significantly during the study (see Fig. 1). The mean exercise time was 9:41 min:s (range 5:34-15:27). The energy efficiency at lower work- loads, $50 \mathrm{~W}$, increased significantly (from $0.16 \%$ [0.03] to $0.18 \%$ [0.02], $p=0.033$ ) during treatment, whereas efficiency at higher workloads was unchanged. Overall, the participants' maximum load until exhaustion was low. However, in $66 \%$ of the tests, the participants reached 
their anaerobic threshold defined as an increasing respiratory quotient (VO2/VCO2) surpassing 1.0. There was no significant change in perceived tiredness (BORG scale) at 50 or $75 \mathrm{~W}$, during the study.

Body Weight Correlations with REE, Physical

Activity, or Exercise Performance, within and between

Individuals

Within-person correlation coefficients with body weight as the dependent variable were calculated for parameters with possible influence on body weight changes (Table 2). The significant (negative) within-person correlation between REE and body weight suggests that a decrease in REE correlates to increase in body weight in an individual. The significant (negative) between-investigation correlation between $\mathrm{fT} 3$ and body weight means that during the study, high level of fT3 correlated to low body weight.

The EFFE $50 \mathrm{~W}$ correlated significantly between-investigations, suggesting that low energy efficiency correlates to low body weight. However, changes in efficiency did not correlate to changes in body weight, in the individual. Efficiency at $50 \mathrm{~W}$ at baseline did not correlate to fT3 at baseline or changes in body weight, during the study.

\section{Discussion}

This study shows that despite quite large changes in thyroid hormone levels; the impact on daily physical function level was limited in sedentary patients treated for marked hyperthyroidism. The changes in physical performance within 1 year were only affected at lower work intensities, and the most pronounced change was in REE. Changes in body weight were evident with a mean increase of $5.2 \mathrm{~kg}$, but with rather large individual differences, as changes ranged from $8.9 \mathrm{~kg}$ weight loss to 15.1 $\mathrm{kg}$ weight gain during 1 year of treatment. No significant changes in body composition occurred, and yet in relative changes, the lean mass component was the most affected (7.5\% compared to $0.6 \%$ for bone mass and $3.2 \%$ for fat mass), and the level of hyperthyroidism at baseline (fT3) predicted $78 \%$ of the increase in lean mass, during the year. A predominant increase in the lean mass compartment, during treatment of hyperthyroidism, has previously been shown by Lonn and coworkers [3]. In this study, DXA was used to measure body composition and the fat mass also increased, although at a later time point than the increase in lean mass. With the use of bioimped-
Table 2. Within-person and between-investigation correlations between body weight and various parameters of potential influence on body weight

\begin{tabular}{lrrrl}
\hline $\begin{array}{l}\text { Body weight } \\
\text { correlation with: }\end{array}$ & $\begin{array}{l}\text { Within- } \\
\text { person } \\
\text { correlation }\end{array}$ & $p$ value & $\begin{array}{l}\text { Between- } \\
\text { investigation } \\
\text { correlation }\end{array}$ & $p$ value \\
\hline REE & -0.390 & $\mathbf{< 0 . 0 0 1}$ & -0.620 & 0.14 \\
REE/expected, \% & -0.565 & $\mathbf{0 . 0 0 1}$ & -0.724 & 0.066 \\
REE/kg & -0.661 & $<\mathbf{0 . 0 0 1}$ & -0.830 & $\mathbf{0 . 0 2 1}$ \\
Daily activity, METs/day & 0.106 & 0.395 & 0.287 & 0.53 \\
Pedometer, steps/day & -0.149 & 0.219 & -0.235 & 0.61 \\
Efficiency 50 W, \% & 0.180 & 0.144 & 0.791 & $\mathbf{0 . 0 3 3}$ \\
Efficiency 75 W, \% & 0.123 & 0.328 & 0.529 & 0.22 \\
Efficiency max load, \% & 0.104 & 0.403 & 0.384 & 0.40 \\
fT3, pmol/L & -0.539 & $<\mathbf{0 . 0 0 1}$ & -0.785 & $\mathbf{0 . 0 3 6}$ \\
\end{tabular}

Within-person correlation examine if change in 1 parameter correlate to change in another parameter in an individual, whereas between-investigation correlations considers whether a high (or low) value in 1 parameter correlates to a high (or low) value in another parameter between individuals. MET, metabolic equivalent; $\mathrm{W}$, watt; fT3, free T3; REE, resting energy expenditure; max, maximum. A $p$ value of $<0.05$ was considered significant.

Table 3. Categories of different physical activity levels, with its corresponding MET values [14], approximate measurement method in this study, and the participants' reporting of time spent in each category

\begin{tabular}{lllll}
\hline Category Description & $\begin{array}{l}\text { MET } \\
\text { value }\end{array}$ & $\begin{array}{l}\text { Measured } \\
\text { equivalent }\end{array}$ & $\begin{array}{l}\text { Amount of time } \\
\text { spent in each } \\
\text { category, h/day; } \\
24 \text { h, \% }\end{array}$ \\
\hline A & Sleeping & 0.9 & REE & $(8.1) ; 33.6$ \\
B & Reading & 1 & REE & $(3.4) ; 14.1$ \\
C & PC working & 1.5 & REE & $(5.3) ; 22.0$ \\
D & Standing & 2 & & $(3.0) ; 12.5$ \\
E & Walking & 3 & 50 W exercise & $(2.0) ; 8.31$ \\
F & Brisk walking & 4 & 75 W exercise & $(1.4) ; 5.81$ \\
G & Gardening & 5 & 75 W exercise & $(0.59) ; 2.45$ \\
H & Exercising & 6 & Max load & $(0.18) ; 0.75$ \\
I & Strenuous exercise & 6 & Max load & $(0.11) ; 0.05$ \\
\hline
\end{tabular}

MET, metabolic equivalent; $W$, watt; REE, resting energy expenditure; max, maximum.

ance, Chng and coworkers found that fat mass only increased after euthyroidism was achieved after 38 months of ATD treatment [19]. Taken together, this may suggest that, during treatment of hyperthyroidism, weight increase is likely and changes in the lean mass compartment 
predominate in the short to medium term, whereas changes in the fat mass occur at a later stage.

When individual changes were considered, body weight correlated to changes in REE, but not to energy efficiency at lower workloads. This finding is in accordance with time spent in the different physical activity levels, as shown in Table 3 . If REE covers approximately the MET values from 0.9 to $1.5 \mathrm{MET}$, around $70 \%$ of the time during an average $24 \mathrm{~h}$ day was spent in this MET interval. Only around $10 \%$ of the time was spent in a MET intensity corresponding to $50 \mathrm{~W}$ (see Table 3 ). The finding of reduced energy efficiency has previously been shown by Kimura and coworkers who found a reduced efficiency of oxygen utilization in work load in the spectrum between 10 and $80 \mathrm{~W}$, in hyperthyroidism compared to euthyroidism [5].

In contrast to previous studies [6,20,21], we did not find reduced exercise capacity with regard to maximum workload achieved, in the untreated versus the treated state. The testing of maximum workload, however, was not a main purpose of the work test. The lack of change in the maximum workload achieved in the untreated compared to the treated patients in our study could thus be due to exhaustion rather than reduced maximum work capacity, as a test for maximum work capacity would optimally have a steeper increase in workload during the test.

There were some dynamics in physical activity levels during the first 4 months, but this leveled out and was not proved better after 1 year of treatment. The measures of physical activity of daily living showed large variation. The within-person and between-person CV in the number of steps per day and the activity questionnaire were $27 / 25$ and $11 / 16 \%$, respectively.

Strength of the study: This study was designed as a prospective repeated measurement study, which enables analyses of variable correlations within individuals. This is important when studying variables with high level of individuality such as body weight. The power in a repeated measurement study is in the number of tests per patient rather than in the number of patients. This study was well powered with regard to the primary end-point, body weight changes. However, some of the negative findings, such as the insignificant increase in lean mass, could be due to lack of power.

Limitations: We focused on energy expenditure. Body weight, however, is a product of the balance between energy expenditure and energy intake. We did not measure energy intake, and this hampers the possibility of a detailed analysis of the total energy flux during treatment of hyperthyroidism. The results found in the present study, however, were not influenced by the absence of an analysis of the total energy flux.

We only included and studied patients with hyperthyroidism who were scheduled to medical antithyroid treatment. The rapid hormonal changes during surgical treatment and the usually more protracted changes during radioiodine treatment may generate different dynamics in body weight changes than we present here. We followed the patient for 1 year; thus, we were not able to consider the long-term impact on body weight in patients treated for hyperthyroidism.

The measures of physical activity of daily living showed large variation, and this increases the risk of missing a true change (statistical type II error).

\section{Conclusion}

This study showed that treatment of hyperthyroidism was associated with marked increases in body weight, in particular the lean mass component, and the weight gained seem to exceed the weight lost prior to diagnosis. In sedentary patients, this increase in body weight correlated to a decrease in REE and only to a negligible extent to changes in energy efficiency and not at all to changes in physical activity level of daily living.

Sedentary patients newly diagnosed with overt hyperthyroidism may, therefore, expect weight gain without improvement (or decline) of their physical capacity after treatment is initiated. This is an important message, as many patients report weight loss, tiredness, and reduced physical capacity at disease onset, and many patients do not automatically expect weight gain following antithyroid treatment. In other words, if no lifestyle changes occur, a marked weight gain is to be expected after 1 year of treatment for hyperthyroidism.

\section{Acknowledgement}

The late Professor Peter Laurberg was the main originator of this study.

\section{Statement of Ethics}

This study was approved by the Regional Ethics Committee in North-Jutland and Viborg County, Denmark. Informed consent was signed by all the participants before entering the study. 


\section{Conflict of Interest Statement}

J.K. has no conflicts of interest to declare. A.C. has received financial aid from Beckett Foundation but declares no conflicts of interest. S.A. has no conflicts of interest to declare.

\section{Funding Sources}

This study was funded by an unrestricted grant from Aalborg University, Denmark.

\section{Author Contributions}

J.K. was involved in the design of this study and carried the practical part of the study through, as well as contributing to writing the manuscript. A.C. was involved in the design of the study and in writing the manuscript. S.A. was involved in the design of this study and in writing the manuscript.

\section{References}

1 De Leo S, Lee SY, Braverman LE. Hyperthyroidism. Lancet. 2016 Aug;388(10047):90618.

2 Kyriacou A, Kyriacou A, Makris KC, Syed AA, Perros P. Weight gain following treatment of hyperthyroidism: a forgotten tale. Clin Obes. 2019 Oct;9(5):e12328.

3 Lonn L, Stenlof K, Ottosson M, Lindroos AK, Nystrom E, Sjostrom L. Body weight and body composition changes after treatment of hyperthyroidism. J Clin Endocrinol Metab. 1998 Dec;83(12):4269-73.

4 Silva JE. The thermogenic effect of thyroid hormone and its clinical implications. Ann Intern Med. 2003;139(3):205-13.

5 Kimura H, Kawagoe Y, Kaneko N, Fessler HE, Hosoda S. Low efficiency of oxygen utilization during exercise in hyperthyroidism. Chest. 1996 Nov;110(5):1264-70.

6 Kahaly G, Hellermann J, Mohr-Kahaly S, Treese N. Impaired cardiopulmonary exercise capacity in patients with hyperthyroidism. Chest. 1996 Jan;109(1):57-61.

7 Kung AWC. Neuromuscular complications of thyrotoxicosis. Clin Endocrinol. 2007; 67(5):645-50.

8 Brennan MD, Powell C, Kaufman KR, Sun PC, Bahn RS, Nair KS. The impact of overt and subclinical hyperthyroidism on skeletal muscle. Thyroid. 2006;16(4):375-80.
9 Simonides WS, van Hardeveld C. Thyroid hormone as a determinant of metabolic and contractile phenotype of skeletal muscle. Thyroid. 2008;18(2):205-16.

10 Jacobsen R, Lundsgaard C, Lorenzen J, Toubro S, Perrild H, Krog-Mikkelsen I, et al. Subnormal energy expenditure: a putative causal factor in the weight gain induced by treatment of hyperthyroidism. Diabetes Obes Metab. $2006 \mathrm{Mar} ; 8(2): 220-7$.

11 Levine JA, Nygren J, Short KR, Nair KS. Effect of hyperthyroidism on spontaneous physical activity and energy expenditure in rats. J Appl Physiol. 2003 Jan;94(1):165-70.

12 Harris JA, Benedict FG. A biometric study of human basal metabolism. Proc Natl Acad Sci U S A. 1918;4(12):370-3.

13 Borg GA. Psychophysical bases of perceived exertion. Med Sci Sports Exerc. 1982;14(5): 377-81.

14 Aadahl M, Jørgensen T. Validation of a new self-report instrument for measuring physical activity. Med Sci Sports Exerc. 2003;35(7): 1196-202.

15 Ainsworth B, Haskell WL, Leon AS, Jacobs DR, Montoye HJ, Sallis JF, et al. Compendium of physical activity. Med Sci Sports Exerc. 1993;25(1):71-80.
16 Harris EK, Cooil BK, Shakarji G, Williams GZ. On the use of statistical models of withinperson variation in long-term studies of healthy individuals. Clin Chem. 1980;26(3): 383-91.

17 Bland JM, Altman DG. Calculating correlation coefficients with repeated observations: Part 1 - correlation within subjects. BMJ. 1995 Feb;310(6977):446.

18 Bland JM, Altman DG. Statistics notes: calculating correlation coefficients with repeated observations: Part 2 - correlation between subjects. BMJ. 1995 Mar;310(6980):633.

19 Chng CL, Lim AY, Tan HC, Kovalik JP, Tham $\mathrm{KW}$, Bee YM, et al. Physiological and metabolic changes during the transition from hyperthyroidism to euthyroidism in graves' disease. Thyroid. 2016 Oct;26(10):1422-30.

20 Irace L, Pergola V, Di Salvo G, Perna B, Tedesco MA, Ricci C, et al. Work capacity and oxygen uptake abnormalities in hyperthyroidism. Minerva Cardioangiol. 2006 Jun; 54(3):355-62.

21 Ben-Dov I, Sietsema KE, Wasserman K. O2 uptake in hyperthyroidism during constant work rate and incremental exercise. Eur J Appl Physiol Occup Physiol. 1991;62(4):2617. 